\title{
Correction to: Physiological Mechanism of Drought-Resistant Rice Coping With Drought Stress
}

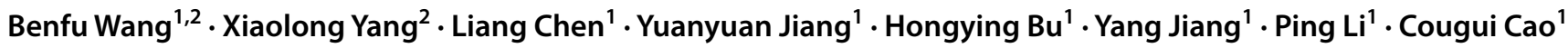

Published online: 24 November 2021

○) Springer Science+Business Media, LLC, part of Springer Nature 2021

\section{Correction to: Journal of Plant Growth Regulation https://doi.org/10.1007/s00344-021-10456-6}

In the original publication of this article, the author Dr. Jianping Cheng was included incorrectly as a co-author. The updated author list is shown in this erratum. All authors agree to this amendment.

The authors would also like to amend their Author Contribution Statement. The amended statement reads:

Cougui Cao and Ping Li conceived the experiments. Xiaolong Yang, Liang Chen, Hongying Bu and Yuanyuan Jiang conducted the experiments and collected the data. Benfu Wang and Yang Jiang analyzed the data and Benfu Wang wrote the manuscript. All authors revised the manuscript.

Finally, the authors would like to include the following Acknowledgement:

Thanks to Jianping Cheng for work in the revising the manuscript of language.

The original article can be found online at https://doi.org/10.1007/ s00344-021-10456-6.

Cougui Cao

ccgui@mail.hzau.edu.cn

Xiaolong Yang

yang8083334@163.com

Liang Chen

1013400479@qq.com

Yuanyuan Jiang

532206230@qq.com

Hongying $\mathrm{Bu}$

764992312@qq.com

Yang Jiang

jiangyang@mail.hzau.edu.cn
Publisher's Note Springer Nature remains neutral with regard to jurisdictional claims in published maps and institutional affiliations.
Ping Li

sleep1022@mail.hzau.edu.cn

1 MOA Key Laboratory of Crop Physiology, Ecology and Cultivation (The Middle Reaches of Yangtze River)/ College of Plant Science and Technology, Huazhong Agricultural University, No.1, Shizishan Street, Hongshan District, Wuhan 430070, Hubei, China

2 Institute of Food Crops, Hubei Academy of Agricultural Sciences, Hubei Key Laboratory of Food Crop Germplasm and Genetic Improvement, Wuhan 430064, Hubei, China 